\title{
The Electrophysiological Signature of Motivational Salience in Mice and Implications for Schizophrenia
}

\author{
Carolin Moessnang ${ }^{1,2,3}$, Ute Habel ${ }^{1,2}$, Frank Schneider ${ }^{1,2}$ and Steven J Siegel ${ }^{*, 3}$ \\ 'Department of Psychiatry, Psychotherapy and Psychosomatics, RWTH Aachen University, Aachen, Germany; ${ }^{2}$ JARA-Translational Brain \\ Medicine, Jülich, Germany; ${ }^{3}$ Department of Psychiatry, Translational Research Laboratory, University of Pennsylvania, Philadelphia, PA, USA
}

\begin{abstract}
According to the aberrant-salience hypothesis, attribution of motivational salience is severely disrupted in patients with schizophrenia. To provide a translational approach for investigating underlying mechanisms, neural correlates of salience attribution were examined in normal mice and in a MK-80 I model of schizophrenia. Electrophysiological responses to standard and deviant tones were assessed in the medial prefrontal cortex (mPFC) using an auditory oddball paradigm. Motivational salience was induced by aversive conditioning to the deviant tone. Analysis of the auditory evoked potential (AEP) showed selective modulation of the late frontal negativity (LFN) by motivational salience, which persisted throughout a 4-week delay. MK-80I, an N-methyl-D-aspartic acid receptor antagonist, abolished this differential response to motivational salience in conditioned mice. In contrast, a pronounced LFN response was observed towards the deviant, ie, perceptually salient tone, in nonconditioned mice. The finding of a selective modulation of a late frontal slow wave suggests increased top-down processing and emotional evaluation of motivationally salient stimuli. In particular, the LFN is discussed as the mouse analog to the human stimulus preceding negativity, which reflects preparatory processes in anticipation of reward or punishment. MK-80I led to a disruption of the normal response in conditioned and nonconditioned mice, including an aberrantly increased LFN in nonconditioned mice. This pattern of 'false-negative' and 'false-positive' responses suggests a degradation of salience attribution, which points to mPFC responses to be relevant for translational research on cognitive alterations in schizophrenia. Neuropsychopharmacology (2012) 37, 2846-2854; doi:I0.1038/npp.2012.156; published online 22 August 2012
\end{abstract}

Keywords: salience; electrophysiology; anticipation; mPFC; schizophrenia; mouse

\section{INTRODUCTION}

Motivational salience of a stimulus refers to the property of capturing attention and driving behavior because of previous associations with reward or punishment. According to the aberrant-salience hypothesis, abnormal attribution of motivational salience is one of the main contributors to both positive and negative symptoms in schizophrenia. Based on converging pharmacological and imaging findings, this theory states that the degradation of the signalto-noise ratio in the striatum results in an impaired representation of motivational salience (Heinz and Schlagenhauf, 2010). As a consequence, patients fail to recognize the predictive property of a stimulus that has previously been associated with reward or punishment, and thus suffer from a reduced ability to discriminate between salient and neutral stimuli. This not only leads to misinterpretation of actually encountered stimuli (ie, false alarms), which might promote the formation of delusions, but also impairs the accurate anticipation of reward and

*Correspondence: Dr SJ Siegel, Department of Psychiatry, University of Pennsylvania, Translational Research Laboratories, $125 \mathrm{~S} 31 \mathrm{St}$, Philadelphia, PA 19104, USA, Tel: + 215573 0278, Fax: + 215573 204I,E-mail: siegels@upenn.edu or siegels@mail.med.upenn.edu Received 7 May 2012; revised 13 July 2012; accepted 25 July 2012 punishment (ie, misses), which might be related to negative symptoms, such as anhedonia and blunted affect (Ziauddeen and Murray, 2010).

Cortical electrophysiological responses in humans and animals suggest the modulation of both early and late components of event-related potentials by emotional and motivationally salient stimuli, as demonstrated by studies using incentive or aversive conditioned stimuli (CS; Baas et al, 2002; Quirk et al, 1995; Tang et al, 2003). Early effects, such as the potentiation of the $\mathrm{P} 1-\mathrm{N} 1$ complex, are exogenously triggered and might reflect modality-specific tuning of the underlying sensory cortex, which allows enhanced encoding of the salient stimulus (Weinberger, 2004). Late components are associated with higher-order, supra-sensory processes. A frontal negative slow wave, which is referred to as stimulus preceding negativity (SPN), is associated with the anticipation of reward and punishment in humans (Brunia et al, 2011), and can thus be regarded as an electrophysiological index of motivational salience. No corresponding late component has been described in rodents so far. However, a cortical slow wave was reported in the auditory cortex of rats, which was interpreted as CS anticipation (Quirk et al, 1997).

This study investigates the electrophysiological correlates of motivational salience and their alteration by a schizophrenia-like state in mice. It has repeatedly been shown that 
electroencephalogram (EEG) recordings in mice show similarities to the human EEG, which makes it attractive to be used in animal models of diseases (Amann et al, 2010). To isolate the specific impact of motivational as compared with perceptual salience (change in stimulus characteristics, eg, 6 $v s 9 \mathrm{kHz}$ ) on neural processes, a complex environment of salience was generated using an oddball paradigm. Perceptual salience was implemented as the pop-out effect of a rare tone embedded in a series of standard tones. Motivational salience was induced by previous coupling of the rare tone (CS) with an aversive event (foot shock; unconditioned stimulus, US; Figure 1). EEG was then recorded in the medial prefrontal cortex (mPFC), which is defined as the prelimbic subdivision of the mouse prefrontal cortex (DeVito et al, 2010; Guldin et al, 1981). To investigate the cortical response to motivational salience as a function of time, recordings were performed immediately after conditioning and following a 4-week delay. In another condition, a subset of mice was additionally treated with the $N$-methyl-D-aspartic acid receptor (NMDAR) antagonist MK-801 to pharmacologically induce a schizophrenia-like state during EEG recordings (Kantrowitz and Javitt, 2010). We hypothesized that mice would show a similar EEG pattern compared with humans in response to salient stimuli and can thus be used as a model to study salience attribution in schizophrenia. More precisely, we expected a specific impact of motivationally salient stimuli on late auditory evoked potential (AEP) components, analogously to the human SPN response. We also anticipated that the induction of a schizophrenialike state by NMDAR disruption should mimic aberrant salience processing in schizophrenia, with reduced response to motivationally salient stimuli, and aberrantly increased response to neutral stimuli.

\section{MATERIALS AND METHODS}

\section{Animals}

Eighteen male C57BL/6Hsd (BL6) mice were obtained at 7-8 weeks of age from Jackson Labs (Bar Harbor, ME). After a 7-day acclimation phase, mice underwent surgery for stereotactic electrode implantation, and were single housed for the remainder of the study. All subjects were maintained in a standard $12 \mathrm{~h}$ light/dark cycle with free access to food and water. The experimental protocols were performed in accordance with the University Laboratory Animal Resources guidelines and were approved by the Institutional Animal Care and Use Committee. Experiments were conducted during the light phase from 0800 to 0000 hours and 1500 to 1900 hours.

\section{Surgery}

Electrode assemblies (PlasticsOne, Roanoke, VA) for nonanesthetized recording of AEPs were stereotactically implanted as previously reported (Amann et al, 2009). Unipolar recording electrodes (PlasticsOne) were placed into the right mPFC $-2.3 \mathrm{~mm}$ anterior, $0.5 \mathrm{~mm}$ lateral, and $1.8 \mathrm{~mm}$ deep to Bregma. Negative and ground electrodes were placed in transversal direction ( 1.5 and $2.5 \mathrm{~mm}$ lateral, respectively) onto the surface of the ipsilateral neocortex $(0.8 \mathrm{~mm}$ deep). Signals recorded with this electrode configuration will emphasis neocortical activity in the medial portion of the PFC (Franklin and Paxinos, 1997). The electrode pedestal was secured to the skull using ethyl cyanoacrylate (Loctite, Henkel, Germany) and dental cement (Ortho Jet, Lang Dental, Wheeling, Illinois). All animals were allowed to recover 1 week. a

Timeline of training sessions and group-specific assignment of events across sessions

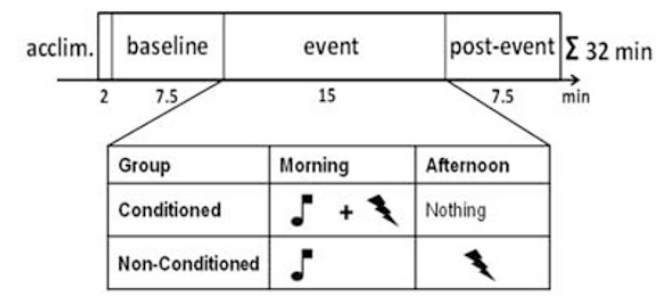

b Training and subsequent test in modified context

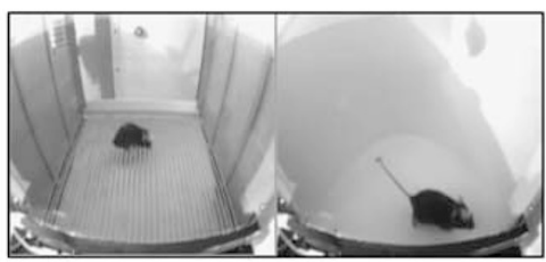

C

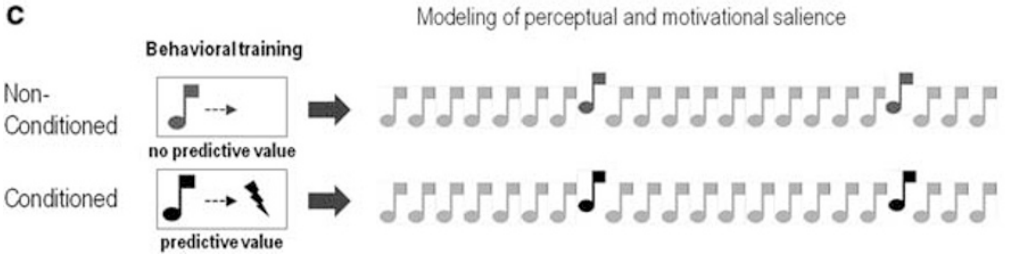

Perceptual salience of the deviant tone

Perceptual and motivational salience of the deviant tone

Figure I (a) Training and test sessions of the cued conditioning paradigm consisted of an acclimation phase, followed by a baseline phase, an event phase during which shocks and/or tones were delivered, and post-event phase. Mice assigned to the conditioned group $(n=9)$ received tones paired with shocks in the morning session, but were placed into the chamber without any stimulation in the afternoon session. Nonconditioned mice $(n=9)$ received only tones in the morning, and only shocks in the afternoon session. (b) Behavioral testing was performed in a modified context. For this purpose, grids were covered by an evenly textured, hard plastic floor. Both floor and walls were reconstructed by card-stock paper, transforming the chamber into a whitecolored semi-circle. The olfactory context was changed using cotton balls soaked with orange concentrate (McCormick, Sparks, MD). (c) Perceptual salience was modeled as the pop-out effect of a deviant tone $(9 \mathrm{kHz})$ in a series of standard tones $(6 \mathrm{kHz})$. Previous aversive conditioning endowed the deviant with additional motivational salience. 


\section{Cued Fear Conditioning}

Behavioral training and test. The experimental protocol started on day 1, when mice were carefully handled and familiarized to the testing and recording environments on three consecutive days (day 1-3, Table 1). On days 4-6, conditioning was performed in ventilated, sound-attenuated chambers (Med Associates, St Albans, VT, USA), which consisted of a metal grid floor, two stainless steel side walls, and a Plexiglas door, back, and ceiling. Training was performed twice a day on day 4 and 6 (Table 1), with explicit pairing of tones and shocks in the experimental group, and explicit un-pairing in nonconditioned mice (Figure 1a). Auditory stimuli $(n=90)$ were delivered by wall-mounted speakers and consisted of $9000 \mathrm{~Hz}$ tones of $10 \mathrm{~ms}$ duration and mean interstimulus interval (ISI) of $10 \mathrm{~s}(8.5-11.5 \mathrm{~s}$, jittered in $500 \mathrm{~ms}$ steps). In the paired condition, tones were pseudorandomly interspersed with scrambled $65-\mathrm{mA}$ foot shocks $(n=9)$ of $500 \mathrm{~ms}$ duration, which were delivered $500 \mathrm{~ms}$ after the onset of the preceding tone. For nonconditioned mice, the only-tone and only-shock conditions consisted of identical sequences of either tones or shocks. Equal perception of shocks in both groups was verified by comparing freezing levels during and following presentation of shocks in the first training session on day $4(p>0.05)$. On day 5 , mice were tested for conditional freezing in response to the CS in a modified context, including different shape, color, texture, and smell of the chamber (Figure 1b). Each animal was tested in a different chamber than their individual training chamber, and exposed to the identical 'onlytone' session as used during training in nonconditioned mice. Mice were videotaped throughout all sessions, and freezing was assessed as percent time spent immobile.

Behavioral analysis. Freezing was quantified in naive mice before any stimulation ('pre-shock baseline'), ie, baseline of session1, day 4, and in trained mice during the test session in the modified context prior ('baseline'), during ('event phase'), and following ('post-event phase') the presentation of the cue. Increased freezing to tones in the modified context reflects CS-dependent fear in conditioned mice. Freezing values were entered into a $2 \times 2$-way repeated measures analysis of variance (rmANOVA), with within-subject factor 'time point' (pre-shock baseline $v s$ baseline $v s$ event phase $v s$ post-event phase) and between-subject factor 'group' (conditioned $v s$ nonconditioned). Post-hoc tests were calculated as matched, two-sample $t$-tests for within-subject comparisons, and as independent samples $t$-tests for betweensubject comparisons (only within conditions). Degrees of freedom are reported in subscript to each $t$ statistic. The Bonferroni correction for multiple comparisons was applied, with $\alpha_{\text {crit }}$ resulting from division of $\alpha=0.05$ by the number of calculated, independent tests. Effect sizes (Cohen's d) were calculated for all post-hoc tests. Statistical calculations were performed with SPSS 17.0 (SPSS, Chicago, IL).

\section{Electrophysiological Recordings}

Recording. Electrophysiological data were assessed before (day 2 and 3) and following fear conditioning (day 7-9, and after a 4-week delay, Table 1) to quantify effects of motivational salience on AEPs. Recordings were performed inside a Faraday electrical isolation cage with background white noise of $70 \mathrm{~dB}$. Electrode pedestals were connected to a $30-\mathrm{cm}$ tripolar electrode cable, which allowed unrestrained movement in the recording chamber. EEG recording and stimulus generation were controlled by Micro1401 hardware and Spike 5 software (Cambridge Electronic Design, Cambridge, UK). EEG data were digitized at a rate of $1667 \mathrm{~Hz}$ with a $1-500-\mathrm{Hz}$ bandpass filter. Tones were delivered through speakers attached to the cage top and the sound pressure was calibrated to $85 \mathrm{~dB}$ inside the cage. Each recording session was preceded by a 10-min acclimation phase.

Pre-exposure to the standard tones. During the morning session of day 2 and 3, mice were exposed to a series of $6 \mathrm{kHz}$ tones $(n=1000$, duration $=10 \mathrm{~ms}$, ISI $=500 \mathrm{~ms})$. This pre-exposure was expected to induce latent inhibition,

Table I Experimental Protocol

\begin{tabular}{|c|c|c|c|c|c|c|c|}
\hline \multicolumn{4}{|c|}{ Behavioral protocol } & \multicolumn{4}{|c|}{$\begin{array}{l}\text { EEG protocol } \\
\text { Oddball: tone I as standard, tone } 2 \text { as deviant }\end{array}$} \\
\hline Day I-3 & Day 4 & Day 5 & Day 6 & Day 7 & Day 8 & Day 9 & 4 Weeks later \\
\hline \multicolumn{8}{|l|}{ Morning session } \\
\hline Handling/EEG I and I| & Training & Test & Training & EEG III & EEG IV & EEG V & EEG VI \\
\hline Pre-exposure to tone I & $\begin{array}{l}\text { Cued fear conditioning } \\
\text { to tone } 2\end{array}$ & $\begin{array}{l}\text { Freezing to tone } \\
2 \text { in modified context }\end{array}$ & $\begin{array}{l}\text { Cued fear conditioning } \\
\text { to tone } 2\end{array}$ & Oddball & $\begin{array}{l}\text { MK-80 I or } \\
\text { saline injection }\end{array}$ & $\begin{array}{l}\text { MK-80I or } \\
\text { saline injection }\end{array}$ & Oddball \\
\hline \multicolumn{8}{|l|}{ Afternoon session } \\
\hline \multirow[t]{2}{*}{ Handling } & Training & & Training & & & & \\
\hline & $\begin{array}{l}\text { Cued fear } \\
\text { conditioning to tone } 2\end{array}$ & & $\begin{array}{l}\text { Cued fear } \\
\text { conditioning to tone } 2\end{array}$ & & & & \\
\hline
\end{tabular}

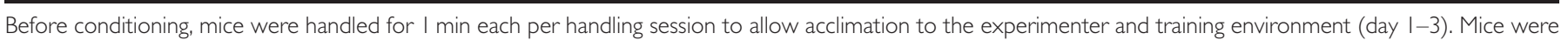

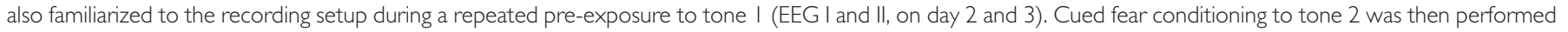
(day 4), and tested $24 \mathrm{~h}$ later in a modified context (day 5). Training was repeated on day 6 to stabilize learning. AEPs were assessed using an auditory oddball

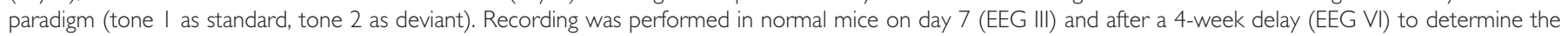

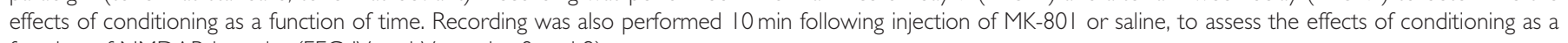
function of NMDAR integrity (EEG IV and $V$, on day 8 and 9). 
which prevents generalization of the conditioned fear response (Lubow, 1973). Thus, only deviant tones (ie, CS) should be associated with electric shocks (US) in the oddball recordings following fear conditioning. In addition, peak amplitudes of AEPs recorded on day 3 were compared between groups and equal auditory processing was confirmed (see supplementary material). All pre-exposure recordings were performed in the animal's home cage.

Recording of AEPs during the oddball paradigm. The oddball paradigm consisted of a series of $10-\mathrm{ms} 6 \mathrm{kHz}$ standard tones interspersed with $10-\mathrm{ms} 9 \mathrm{kHz}$ deviant tones. The ISI between two consecutive tones was fixed at $500 \mathrm{~ms}$ and thus identical to the presentation rate of standards during the pre-exposure. In total, 70 deviant tones were presented at a mean interval of $10 \mathrm{~s}(8.5-11.5 \mathrm{~s}$, jittered in $500 \mathrm{~ms}$ steps), which is identical to the presentation rate of the CS used during fear conditioning. On day 5 and 6 , a subset of mice $(n=12)$ were injected with either $0.25 \mathrm{mg} / \mathrm{kg}$ MK-801 (Sigma-Aldrich, St Louis, MO) dissolved in saline, or an equal amount of saline $10 \mathrm{~min}$ before recording. Injections of drug or saline were counter-balanced across groups and days. Owing to an expected drug-induced increase in EEG signal variance, the number of deviant tones was increased to 225. All oddball recordings were preformed in a neutral context (ie, not in the animal's home cage) to exclude potential contextual confounds.

Preprocessing of electrophysiological data and parameter extraction. EEG data were analyzed using EEGLAB (Delorme and Makeig, 2004). Preprocessing encompassed conditionwise epoching around stimulus onset at $t=0$ from -200 to $400 \mathrm{~ms}$, baseline correction, and application of an individually adjusted epoch rejection criterion (ie, rejection of epochs surpassing two times the SD of the individual amplitude distribution). Regarding the oddball paradigm, only standards preceding a deviant were included to create averages with an equal number of trials in each condition. Owing to poor signal quality, three mice were excluded from EEG analysis of the oddball without pharmacological intervention (day 7 and 4 weeks later). Two mice were excluded from the oddball with pharmacological intervention (day 8 and 9). For AEP analysis, condition-wise average waveforms were calculated for each mouse, and amplitude and latency values were extracted as the minimum or maximum value for the P20 (10-30 ms), N40 (30-60 ms), P3 $(50-200 \mathrm{~ms})$, and the late negative component (late frontal negativity, (LFN); 200-400 ms).

Statistical analysis of electrophysiological data. To analyze the effects of salience as a function of time, each ERP measure obtained from the oddball paradigm without pharmacological intervention was entered into a $2 \times 2 \times$ 2-way rmANOVA, with within-subject factors 'tone' (deviant $v s$ standard) and 'time' (EEG III $v s$ EEG VI), and between-subject factor 'group' (conditioned vs nonconditioned). For analyzing the effect of NMDAR dysfunction on salience processing, ERP measures obtained from the oddball paradigm with pharmacological intervention were analyzed using a $2 \times 2 \times 2$-way rmANOVA, with within-subject factors 'tone' (deviant $v s$ standard) and 'drug' (MK-801 vs saline), and between-subject factor 'group' (conditioned $v s$ nonconditioned). As group differences were hypothesized in response to the deviant tone, analyses focused on group $\times$ tone interactions. Post-hoc tests were performed as outlined above. All mean values are presented in Supplementary Table S1.

\section{RESULTS}

\section{Freezing Behavior}

Analysis of freezing revealed a highly significant group $x$ time point interaction $\left(\mathrm{F}_{[3,48]}=17.120, p<0.001\right.$; Supplementary Figure S1). Post-hoc analyses $\left(\alpha_{\text {crit }}=0.003\right)$ confirm CS-induced freezing in the conditioned group, which is significant for the between-groups (conditioned $v s$ nonconditioned: $t_{[16]}=$ $3.934, p<0.001, d=1.94)$ as well as within-group comparisons (pre-shock baseline $v s$ event phase: $t_{[8]}=7.582, p<0.001$, $d=3.25$; baseline $v s$ event phase: $t_{[8]}=9.486, p<0.001$, $d=3.02$; post-event $v s$ event phase: $t_{[8]}=12.840, p=$ $<0.001, d=3.61)$. Crucially, nonconditioned mice demonstrated no differences in freezing across time points.

\section{ERP Results-Salience as a Function of Time}

An overview of rmANOVA results for all peaks is given in Table 2. A significant effect of motivational salience as indicated by a group $\times$ tone interaction was found for LFN amplitude $\left(\mathrm{F}_{[1,13]}=20.231, p=0.001\right)$. In contrast, no peak yielded a three-way interaction of the factors 'tone', 'group', and 'time'. Post-hoc tests $\left(\alpha_{\text {crit }}=0.012\right)$ were therefore calculated over peak values collapsed over both sessions, and corresponding waveforms are depicted in Figure 2. Amplitude of the LFN was significantly increased in conditioned mice subsequent to presentation of the deviant tone in both between- $\left(t_{[13]}=4.485, p=0.001, d=2.37\right)$ and within-group comparisons $\left(t_{[7]}=6.155, p<0.001, d=2.56\right)$. These differential effects were absent in nonconditioned mice (LFN amplitude: $t_{[6]}=0.875, p=0.42, d=0.43$ ).

\section{ERP Results-Salience as a Function of NMDA Integrity}

Following administration of MK-801, the effect of NMDARmediated glutamate disruption on salience attribution is revealed as three-way interaction of the factors 'tone', 'drug', and 'group'. This effect was present for the amplitude of the LFN $\left(\mathrm{F}_{[1,8]}=8.508, p=0.019\right.$; Figure 3$)$. A main effect of 'drug' was evident in all ERP measures except for P3 amplitude and N40 latency, indicating an overall adverse effect of NMDAR disruption (Table 2). Consistent with results of the former analysis, post-hoc comparisons $\left(\alpha_{\text {crit }}=\right.$ 0.004) on LFN amplitude following saline injection showed an increased response to the deviant in conditioned mice $\left(t_{[4]}=6.089, p=0.004, d=1.93\right)$, which, however, did not survive correction for multiple comparisons in the betweengroup comparison $\left(t_{[8]}=3.250, p=0.012, d=2.05\right)$. Selectivity for the deviant tone was abolished following injection of MK-801 (between: $t_{[8]}=1.190, p=0.268, d=0.7$; within: $\left.t_{[4]}=1.926, p=0.126, d=1.18\right)$. Within-group comparisons in nonconditioned mice indicated the emergence of an increased response to deviants after MK-801 $\left(t_{[4]}=2.720\right.$, $p=0.053, d=2.01$; not surviving correction for multiple 
Table 2 Overview of Three-way rmANOVA Results Calculated for Each ERP Measure of the Oddball Paradigms with and without Pharmacological Intervention

Within-subject factors 'tone' (standard vs deviant), 'time' (EEG III vs EEG VI), between-subject factor 'group' (conditioned vs nonconditioned)
Within-subject factors 'tone' (standard vs deviant), 'drug' (MK80 I vs saline), between-subject factor 'group' (conditioned vs nonconditioned)

\begin{tabular}{|c|c|c|c|c|}
\hline & Amplitude & Latency & Amplitude & Latency \\
\hline N40 & ME time $(p=0.043)$ & & ME drug $(p=0.00 I)$ & NS \\
\hline LFN & $\begin{array}{l}\text { IA tone } \times \text { group }(p=0.00 I), \text { ME tone } \\
(p<0.00 \mid) \text {, ME group }(p=0.006)\end{array}$ & & $\begin{array}{l}\text { IA tone } \times \text { drug } \times \text { group } \\
(p=0.019), \text { ME tone }(p=0.00 \mathrm{I})\end{array}$ & $\begin{array}{l}\text { IA tone } \times \text { group } \\
(p=0.004), \text { ME drug }(p=0.044)\end{array}$ \\
\hline
\end{tabular}

Depicted are significant main effects (ME) and interactions (IA) with corresponding $P$-value $(\alpha=0.05)$ of the F-test. Only effects of motivational salience as reflected by group $\times$ tone and group $\times$ tone $\times$ drug interactions (printed in italics) were considered in the main text.

$a$ Electrode location in mPFC

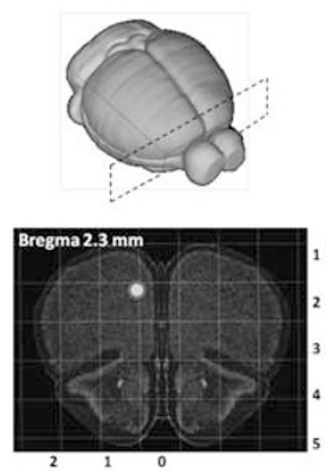

b ERP responses in conditioned and non-conditioned mice
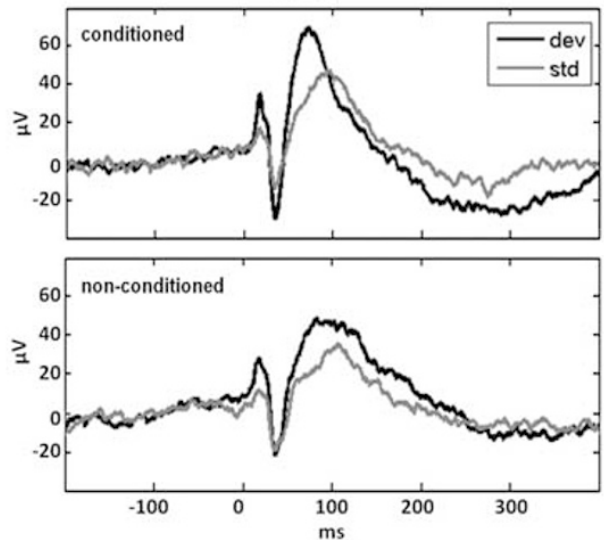

C LFN mean amplitude $( \pm S D)$

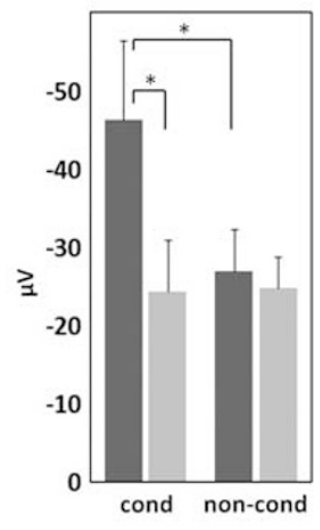

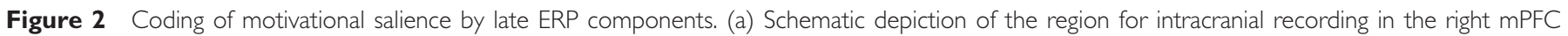

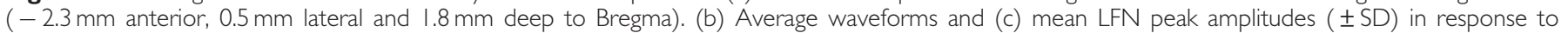

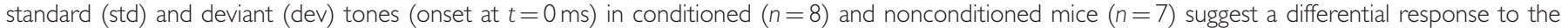

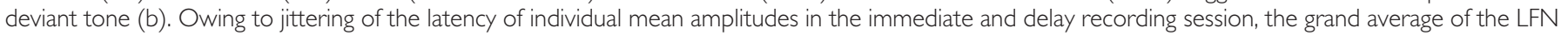
amplitude is attenuated in the average waveform depiction. ${ }^{*} p_{\text {corr }}<0.05$

a

ERP responses to standard and deviant following administration of MK-801 or saline

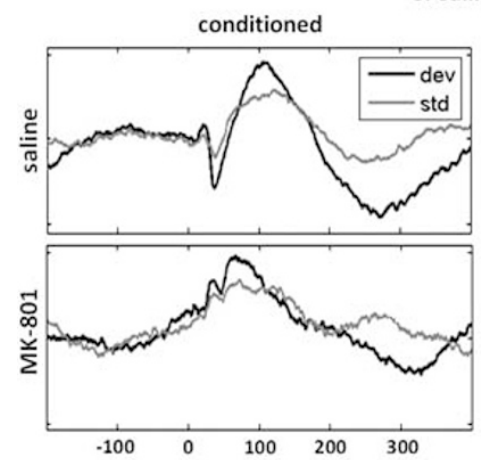

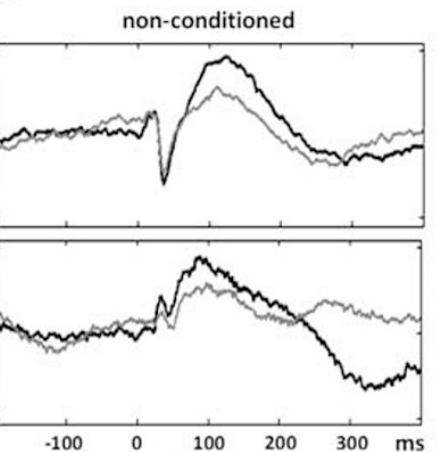

Mean LFN amplitude ( \pm SD) as a function of group, drug and tone

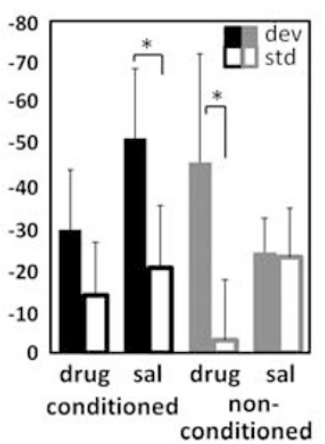

Figure 3 Disruption of salience attribution by NMDAR antagonism. Average waveforms (a) in response to deviants (dev) and standards (std) in conditioned $(n=5)$ and nonconditioned mice $(n=5)$ following injection of saline or $0.25 \mathrm{mg} / \mathrm{kg}$ MK-80 I. Note the resemblance of the late component (LFN) in conditioned mice treated with saline (sal) and the LFN in nonconditioned mice treated with MK-80I. Mean amplitude ( \pm SD) of the LFN shows decreased discrimination between standard and deviant in conditioned mice, but aberrantly increased discrimination in nonconditioned mice following administration of MK-80I (drug). * Puncorr $<0.05$ 
comparisons), which mimicked the 'normal' response of conditioned mice after saline.

\section{DISCUSSION}

In this study, the impact of motivational salience on electrophysiological measures was investigated in normal mice and in the MK-801 model of schizophrenia. Motivational salience was successfully generated using cued fear conditioning of an auditory stimulus. Subsequent prefrontal EEG recordings revealed selective modulation of the LFN, a late frontal slow wave, by motivational salience, an effect that was stable over a 4 -week delay. NMDAR disruption following administration of the pharmacological agent MK801 led to a disruption of the salience-specific LFN effect. More precisely, the LFN response in conditioned mice was highly reduced, leading to a loss of differential response to motivationally salient and neutral tones. In contrast, the LFN response in nonconditioned mice mimicked LFN responses of normal conditioned mice, with significantly increased LFN amplitudes to a motivationally neutral, but perceptually salient tone. Under the assumption that the LFN corresponds to an index of motivational salience, this pattern is consistent with the aberrant-salience hypothesis of schizophrenia, as the induction of a schizophrenia-like state resulted in 'false-negatives' and 'false-positives' in conditioned mice and nonconditioned mice, respectively.

\section{LFN and Stimulus-preceding Negativity}

Using an oddball paradigm with conditioned and nonconditioned deviant tones, we aimed at separating the effects of motivational salience from those of perceptual salience. While the latter primarily relies on bottom-up processing of perceptually outstanding features, the former mainly involves top-down processing and is inherently associated with the activation of appetitive or inhibitory motivational systems (Wynn et al, 2010). The present data are consistent with this notion as only a late frontal slow wave was modulated by motivational salience. The absence of a significant impact of time suggests that there is a robust memory for motivational salience, similar to what has been reported for conditioned responses in general (Bouton, 1994). Although direct comparisons across species are difficult, this slow wave bears intriguing resemblance to a cortical slow wave reported in threat-of-shock experiments in humans, in which subjects anticipated the possible occurrence of an electric shock (Baas et al, 2002). This socalled SPN is reliably elicited in anticipation of motivationally relevant stimuli, such as evocative photos (Poli et al, 2007), aversive noise (Kotani et al, 2001), monetary rewards, (Ohgami et al, 2006) and electrical shocks (Babiloni et al, 2007; Bocker et al, 2001). Cortical downstream targets of the ventral attention and reward system, including MPFC and ACC, have been suggested as generators of the human SPN (van Boxtel and Böcker, 2004). It has even been argued that the SPN is the ultimate cortical consequence of the proposed temporal difference error (TDE; Brunia et al, 2011), which is generated in tegmental neurons in response to motivationally salient stimuli (Schultz et al, 1997). Independent of its generators, the SPN is thought to represent the
EEG-recorded manifestation of the lowering of threshold in wide-spread cortical areas to prepare processing of the anticipated reward or punishment (Brunia, 1993). Under the assumption that basic signaling principles associated with reward and motivation are shared across species, such as the TDE (Rolls et al, 2008; Schultz et al, 2000) or corticostriatolimbic circuitries (Berridge and Kringelbach, 2008; Haber and Knutson, 2010), the late negativity measured in the mPFC of conditioned mice might reflect analogous anticipatory processes in response to motivational salience.

\section{Motivational Salience as a Function of NMDAR Signaling Integrity}

Administration of NMDAR antagonists is a common approach to model schizophrenia-like symptoms in animals (Xi et al, 2009) and humans (Kantrowitz and Javitt, 2010). According to the glutamate hypothesis of schizophrenia, NMDAR hypofunction results in an imbalance of glutamatergic and GABAergic neurotransmission, which leads to wide-ranging sensory, behavioral, and cognitive disturbances. The mPFC has been shown to be one of the primary targets of systemic NMDAR antagonist administration in animals (Jackson et al, 2004) and humans (Breier et al, 1997), which seems to be mediated by subcortical thalamic and limbic structures (Kiss et al, 2011). Another study showed a widespread thalamic and cortical activation after NMDA-R blockade (Santana et al, 2011). In addition to thalamic inputs, NMDA-R blockade in PFC may also contribute (Homayoun and Moghaddam, 2007). The dysregulation of the prefrontal cortex interferes with various corticalstriatal circuits, which directly (Javitt, 2007) and indirectly (Murase et al, 1993; Sesack and Carr, 2002) affect dopamine signaling in the striatum, leading to disturbed salience attribution. Our results support the hypothesis that MK-801 alters processing of motivational salience as reflected by amplitude changes of the LFN, with attenuated responses to motivationally salient events in conditioned mice ('false negatives'), and exaggerated responses to perceptually salient, but motivationally neutral events in nonconditioned mice ('false positives'). This finding is all the more meaningful as it occurs on a background of an overall reduction of AEP amplitudes, which is a common effect of NMDA antagonists on auditory ERPs (Saunders et al, 2012). If the LFN represents anticipatory and preparatory processes, our findings imply that MK-801 ultimately led to inadequate anticipation of the aversive US, ie, aberrant salience attribution. This is consistent with observations of impaired anticipation of emotionally relevant stimuli in patients with schizophrenia. A well-known example is the dissociation between impaired anticipation of reward (ie, anticipatory pleasure) and relatively intact hedonic experience (ie, consummatory pleasure), a phenomenon that has been linked to anhedonia (Gard et al, 2006). Numerous imaging studies reported abnormal neural responses in the reward system of patients with schizophrenia during learning and reward anticipation, with blunted response to salient, and aberrantly increased response to neutral stimuli (Ziauddeen and Murray, 2010). The same applies to the limbic system and emotional stimuli (Habel et al, 2010; Seiferth et al, 2009). The functional significance of aberrant salience attribution is reflected by the correlation with positive (Roiser et al, 2009; Schlagenhauf 
et al, 2009) and negative (Waltz et al, 2009) symptoms, and the partial reversal by antipsychotic medication (Juckel et al, 2006). Complementary to these findings, EEG studies have shown intact early- and mid-latency ERP responses during affective processing, but a reduced SPN response in patients with schizophrenia during anticipation of reward or punishment (Horan et al, 2010; Wynn et al, 2010). An alternate explanation of the effects of MK-801 on processing of motivational salience is interference with mnemonic processes (Amann et al, 2009). In this study, injection of MK-801 might have led to an inability to retrieve salience information in conditioned mice, which resulted in a reduced LFN response. Although interference effects with memory retrieval are more difficult to reconcile with the finding of an increased LFN response in nonconditioned mice, mnemonic alterations might contribute to abnormal salience processing in our MK-801 treated mice, and more generally in patients with schizophrenia. The present results on motivational salience attribution in mice raise the question about their potential impact for gaining a better understanding of diverse symptoms relating to aberrant salience attribution in schizophrenia, such as anhedonia, motivational deficits, or delusional thinking. As our data suggest that the mouse LFN is an electrophysiological marker of motivational salience, comparable to the human SPN, animal models offer a possibility to study a wide range of environmental and genetic determinants of normal and disturbed salience attribution. These findings can in turn inform the development and evaluation of therapeutic strategies in schizophrenia for abnormalities in salience attribution, which is not addressed by current therapies as evidenced by persistent negative symptoms.

\section{Limitations}

A major limitation of this study is the small sample size of $n=5$ mice per group when investigating effects of MK-801 (ie, EEG recording with pharmacological intervention). Along with a relatively high dose of MK-801, effects might have been obscured by increased variance of the electrophysiological signal. However, as the reduction of statistical power bears the risk of type II errors, this drawback does not invalidate our main finding of a reversed SPN response in conditioned and nonconditioned mice. In addition, inbred mouse strains are highly homogenous, which allow the generalization of findings obtained in smaller samples. Finally, effect sizes reported in this study, which range from $d=0.7$ in between-group to $d=2.05$ in within-group comparisons for EEG recordings with MK-801, are comparable to, and even higher than effect sizes reported in human EEG studies. For instance, the comparison of P3 amplitude, which is another instance of long-latency endogenous AEP components, between patients with schizophrenia $(n=23)$ and matched controls $(n=22)$ showed an effect size of 0.63 for P3a and 1.26 for P3b, respectively (Turetsky et al, 2009). Similarly, the between-group comparison of SPN responses between patients with schizophrenia $(n=34)$ and matched controls $(n=36)$ yielded an effect size of 0.78 (Wynn et al, 2010).

A second limitation of the current findings is the lack of control conditions, which unambiguously link the LFN to a subsequent motivational stimulus, as it is the case for the human SPN. The waveforms suggest that the LFN response returns to zero at the time of the expected shock delivery (ie, $500 \mathrm{~ms}$ after stimulus onset). The use of increasing time intervals between CS and US might answer the question about the anticipatory nature in future studies. In addition, the use of stimuli, which have been associated with nonmotivational events, might allow a better differentiation between motivational and non-motivational expectation (eg, Engel and Fries, 2010). However, even if the LFN is more a modulation of a CS evoked component than anticipatory to the US, it can still be interpreted as an electrophysiological signature of motivational salience in mice.

\section{Conclusion}

The combined interpretation of the presented data points to similarities between human and mouse electrophysiological coding of motivational salience. More precisely, a frontal negative slow wave bears temporal, functional, and pharmacological correspondence to the human SPN, and is suggestive for a mouse homology of human salience attribution. Although conclusions based on comparison between rodent EEG and human EEG must remain tentative in view of considerable differences between species, these similarities of phenotypes are not only attractive for further exploration of underlying mechanisms, but can also be used for the study and evaluation of potential therapeutic strategies in disease.

\section{ACKNOWLEDGEMENTS}

We thank Valérie Leitman, John Saunders, Robert Lin, Robert Featherstone, Jimmy Suh, Yuling Liang, and Mike Gandal for their support during data acquisition and analysis. This work was supported by the German Research Foundation (DFG, IRTG 1328), Helmholtz Alliance for Mental Health in an Ageing Society (HelMA, HA-215), Interdisciplinary Center for Clinical Research of the Medical Faculty of the RWTH Aachen University (IZKF, N4-4), and the National Institutes of Health (NIH, grant 5R01DA023210-02).

\section{DISCLOSURE}

Steven Siegel reports having received grant support from Eli Lilly, AstraZeneca, NuPathe, and Pfizer that is unrelated to the content of this paper and consulting payments from NuPathe, Merck, Sanofi, and Wyeth that are unrelated to this work. The rest of the authors declare no conflict of interest.

\section{REFERENCES}

Amann LC, Gandal MJ, Halene TB, Ehrlichman RS, White SL, McCarren HS et al (2010). Mouse behavioral endophenotypes for schizophrenia. Brain Res Bull 83: 147-161.

Amann LC, Halene TB, Ehrlichman RS, Luminais SN, Ma N, Abel T et al (2009). Chronic ketamine impairs fear conditioning and produces long-lasting reductions in auditory evoked potentials. Neurobiol Dis 35: 311-317. 
Baas JM, Kenemans JL, Bocker KB, Verbaten MN (2002). Threatinduced cortical processing and startle potentiation. Neuroreport 13: $133-137$

Babiloni C, Brancucci A, Capotosto P, Del Percio C, Romani GL, Arendt-Nielsen L et al (2007). Different modalities of painful somatosensory stimulations affect anticipatory cortical processes: a high-resolution EEG study. Brain Res Bull 71: 475-484.

Berridge KC, Kringelbach ML (2008). Affective neuroscience of pleasure: reward in humans and animals. Psychopharmacology 199: 457-480.

Bocker KB, Baas JM, Kenemans JL, Verbaten MN (2001). Stimuluspreceding negativity induced by fear: a manifestation of affective anticipation. Int J Psychophysiol 43: 77-90.

Bouton ME (1994). Context, ambiguity, and classical conditioning. Curr Directions Psychol Sci 3: 49-53.

Breier A, Malhotra AK, Pinals DA, Weisenfeld NI, Pickar D (1997). Association of ketamine-induced psychosis with focal activation of the prefrontal cortex in healthy volunteers. Am J Psychiatry 154: $805-811$.

Brunia CH (1993). Waiting in readiness: gating in attention and motor preparation. Psychophysiology 30: 327-339.

Brunia CH, Hackley SA, van Boxtel GJ, Kotani Y, Ohgami Y (2011). Waiting to perceive: reward or punishment? Clin Neurophysiol 122: 858-868.

Delorme A, Makeig S (2004). EEGLAB: an open source toolbox for analysis of single-trial EEG dynamics including independent component analysis. J Neurosci Methods 134: 9-21.

DeVito LM, Lykken C, Kanter BR, Eichenbaum H (2010). Prefrontal cortex: role in acquisition of overlapping associations and transitive inference. Learn Mem 17: 161-167.

Engel AK, Fries P (2010). Beta-band oscillations-signalling the status quo? Curr Opin Neurobiol 20: 156-165.

Franklin K, Paxinos G (1997). The Mouse Brain in Stereotaxis Coordinates. Academic Press: San Diego.

Gard DE, Gard MG, Kring AM, John OP (2006). Anticipatory and consummatory components of the experience of pleasure: a scale development study. J Res Personal 40: 1086-1102.

Guldin WO, Pritzel M, Markowitsch HJ (1981). Prefrontal cortex of the mouse defined as cortical projection area of the thalamic mediodorsal nucleus. Brain Behav Evol 19: 93-107.

Haber SN, Knutson B (2010). The reward circuit: linking primate anatomy and human imaging. Neuropsychopharmacology 35: 4-26.

Heinz A, Schlagenhauf F (2010). Dopaminergic dysfunction in schizophrenia: salience attribution revisited. Schizophr Bull 36: 472-485.

Habel U, Chechko N, Pauly K, Koch K, Backes V, Seiferth N et al (2010). Neural correlates of emotion recognition in schizophrenia. Schizophr Res 122: 113-123.

Homayoun H, Moghaddam B (2007). NMDA receptor hypofunction produces opposite effects on prefrontal cortex interneurons and pyramidal neurons. J Neurosci 27: 11496-11500.

Horan WP, Wynn JK, Kring AM, Simons RF, Green MF (2010). Electrophysiological correlates of emotional responding in schizophrenia. J Abnorm Psychol 119: 18-30.

Jackson ME, Homayoun H, Moghaddam B (2004). NMDA receptor hypofunction produces concomitant firing rate potentiation and burst activity reduction in the prefrontal cortex. Proc Natl Acad Sci USA 101: 8467-8472.

Javitt DC (2007). Glutamate and schizophrenia: phencyclidine, $\mathrm{N}$-methyl-D-aspartate receptors, and dopamine-glutamate interactions. Int Rev Neurobiol 78: 69-108.

Juckel G, Schlagenhauf F, Koslowski M, Filonov D, Wustenberg T, Villringer A et al (2006). Dysfunction of ventral striatal reward prediction in schizophrenic patients treated with typical, not atypical, neuroleptics. Psychopharmacology 187: $222-228$
Kantrowitz JT, Javitt DC (2010). N-methyl-d-aspartate (NMDA) receptor dysfunction or dysregulation: the final common pathway on the road to schizophrenia? Brain Res Bull 83: 108-121.

Kiss T, Hoffmann WE, Scott L, Kawabe TT, Milici AJ, Nilsen EA et al (2011). Role of thalamic projection in nmda receptorinduced disruption of cortical slow oscillation and short-term plasticity. Front Psychiatry 2: 14.

Kotani Y, Hiraku S, Suda K, Aihara Y (2001). Effect of positive and negative emotion on stimulus-preceding negativity prior to feedback stimuli. Psychophysiology 38: 873-878.

Lubow RE (1973). Latent inhibition. Psychol Bull 79: 398-407.

Murase S, Grenhoff J, Chouvet G, Gonon FG, Svensson TH (1993). Prefrontal cortex regulates burst firing and transmitter release in rat mesolimbic dopamine neurons studied in vivo. Neurosci Lett 157: 53-56.

Ohgami Y, Kotani Y, Tsukamoto T, Omura K, Inoue Y, Aihara Y et al (2006). Effects of monetary reward and punishment on stimulus-preceding negativity. Psychophysiology 43: 227-236.

Poli S, Sarlo M, Bortoletto M, Buodo G, Palomba D (2007). Stimulus-Preceding Negativity and heart rate changes in anticipation of affective pictures. Int J Psychophysiol 65: 32-39.

Quirk GJ, Armony JL, LeDoux JE (1997). Fear conditioning enhances different temporal components of tone-evoked spike trains in auditory cortex and lateral amygdala. Neuron 19: 613-624.

Quirk GJ, Repa C, LeDoux JE (1995). Fear conditioning enhances short-latency auditory responses of lateral amygdala neurons: parallel recordings in the freely behaving rat. Neuron 15: 1029-1039.

Roiser JP, Stephan KE, den Ouden HE, Barnes TR, Friston KJ, Joyce EM (2009). Do patients with schizophrenia exhibit aberrant salience? Psychol Med 39: 199-209.

Rolls ET, McCabe C, Redoute J (2008). Expected value, reward outcome, and temporal difference error representations in a probabilistic decision task. Cereb Cortex 18: 652-663.

Santana N, Troyano-Rodriguez E, Mengod G, Celada P, Artigas F (2011). Activation of thalamocortical networks by the N-methylD-aspartate receptor antagonist phencyclidine: reversal by clozapine. Biol Psychiatry 69: 918-927.

Saunders JA, Gandal MJ, Siegel SJ (2012). NMDA antagonists recreate signal-to-noise ratio and timing perturbations present in schizophrenia. Neurobiol Dis 46: 93-100.

Schlagenhauf F, Sterzer P, Schmack K, Ballmaier M, Rapp M, Wrase J et al (2009). Reward feedback alterations in unmedicated schizophrenia patients: relevance for delusions. Biol Psychiatry 65: 1032-1039.

Schultz W, Dayan P, Montague PR (1997). A neural substrate of prediction and reward. Science 275: 1593-1599.

Schultz W, Tremblay L, Hollerman JR (2000). Reward processing in primate orbitofrontal cortex and basal ganglia. Cereb Cortex 10: $272-284$.

Seiferth NY, Pauly K, Kellermann T, Shah NJ, Ott G, HerpertzDahlmann B et al (2009). Neuronal correlates of facial emotion discrimination in early onset schizophrenia. Neuropsychopharm 34: 477-487.

Sesack SR, Carr DB (2002). Selective prefrontal cortex inputs to dopamine cells: implications for schizophrenia. Physiol Behav 77: 513-517.

Tang J, Wagner S, Schachner M, Dityatev A, Wotjak CT (2003). Potentiation of amygdaloid and hippocampal auditory-evoked potentials in a discriminatory fear-conditioning task in mice as a function of tone pattern and context. Eur J Neurosci 18: 639-650.

Turetsky BI, Bilker WB, Siegel SJ, Kohler CG, Gur RE (2009). Profile of auditory information-processing deficits in schizophrenia. Psychiatry Res 165: 27-37.

van Boxtel GJM, Böcker KBE (2004). Cortical measures of anticipation. J Psychophysiol 18: 61-76.

Waltz JA, Schweitzer JB, Gold JM, Kurup PK, Ross TJ, Salmeron BJ et al (2009). Patients with schizophrenia have a reduced neural 
response to both unpredictable and predictable primary reinforcers. Neuropsychopharmacology 34: 1567-1577.

Weinberger NM (2004). Specific long-term memory traces in primary auditory cortex. Nat Rev Neurosci 5: 279-290.

Wynn JK, Horan WP, Kring AM, Simons RF, Green MF (2010). Impaired anticipatory event-related potentials in schizophrenia. Int J Psychophysiol 77: 141-149.

Supplementary Information accompanies the paper on the Neuropsychopharmacology website (http://www.nature.com/npp)
Xi D, Zhang W, Wang HX, Stradtman GG, Gao WJ (2009). Dizocilpine (MK-801) induces distinct changes of N-methyl-Daspartic acid receptor subunits in parvalbumin-containing interneurons in young adult rat prefrontal cortex. Int $J$ Neuropsychopharmacol 12: 1395-1408.

Ziauddeen H, Murray GK (2010). The relevance of reward pathways for schizophrenia. Curr Opin Psychiatry 23: 91-96. 